\title{
Uma acetilação eficiente e econômica do 1,2:3,4-di- $O$-isopropilideno- $\alpha$-D-galactopiranose utilizando anidrido acético ativado in situ pela argila montmorilonita K10
}

\author{
(An efficient and cost-effective acetylation of \\ 1,2:3,4-di-O-isopropylidene- $\alpha$-D-galactopyranose using in situ \\ activated acetic anhydride with clay montmorillonite K10)
}

\author{
C.S.Santos ${ }^{1}$, A.R.S.dos Santos ${ }^{2}$, R. O.Silva ${ }^{2}$, J. R. Freitas Filho ${ }^{1}$, J. C. R. Freitas ${ }^{1,2 *}$ \\ ${ }^{I}$ Universidade Federal Rural de Pernambuco, Departamento de Química, Programa de Pós-graduação em \\ Química, R. Dom Manuel de Medeiros s/n, 52171-900, Recife, PE, Brasil \\ ${ }^{2}$ Universidade Federal de Campina Grande, Centro de Educação e Saúde, Programa de Pós-graduação em \\ Ciências Naturais e Biotecnologia, Cuité, PB, Brasil
}

\begin{abstract}
Resumo
A síntese do 6-acetil 1,2:3,4-di- $O$-isopropilideno- $\alpha$-D-galactopiranose a partir da reação de acetilação do 1,2:3,4-di$O$-isopropilideno- $\alpha$-D-galactopiranose utilizando a argila montmorilonita K10 como promotor é descrita. O protocolo é rápido, eficiente, econômico, livre de solvente e quimiosseletivo. O produto foi obtido em excelente rendimento (95\%), curto tempo reacional $(7,5 \mathrm{~min})$, em temperatura ambiente e sem a necessidade de purificação adicional. Adicionalmente, essa argila manteve sua atividade catalítica por três ciclos reacionais sem diminuição de eficiência. Palavras-chave: catálise heterogênea, síntese orgânica, reação de acetilação, carboidratos, montmorilonita.
\end{abstract}

\begin{abstract}
The synthesis of 6-acetyl 1,2:3,4-di-O-isopropylidene- $\alpha$-D-galactopyranose by the acetylation reaction of 1,2:3,4-di$O$-isopropylidene- $\alpha$-D-galactopyranose using the clay montmorillonite K10 as a promoter is described. The protocol is fast, efficient, cost-effective, solvent-free and chemo-selective. The product was obtained in excellent yield (95\%), short reaction time $(7.5 \mathrm{~min})$, at room temperature without the need of further purification. In addition, this clay maintained its catalytic activity for three reaction cycles without decreasing efficiency.
\end{abstract}

Keywords: heterogeneous catalysis, organic synthesis, acetylation reaction, carbohydrates, montmorillonite.

\section{INTRODUÇÃO}

Atualmente as argilas vêm recebendo uma proeminente atenção dos pesquisadores de diferentes áreas do conhecimento, uma vez que estes materiais apresentam inúmeras aplicações nos mais variados setores industriais [1-3]. As argilas podem ser definidas, de maneira simplista, como sendo um material constituído essencialmente por um grupo de minerais $[4,5]$ que apresentam tamanho de partículas inferiores a 2,0 $\mu \mathrm{m}[6]$. Essas pequenas partículas são constituídas essencialmente por silicatos de alumínio, ferro e magnésio hidratados recebendo o nome de argilominerais, sendo classificados de acordo com sua composição química ou sua estrutura cristalina [6]. As argilas são muitas vezes chamadas de layer silicates (silicatos em camadas) ou

*julianocrufino@yahoo.com.br filossilicatos (silicatos em folhas), pelo fato de serem constituídas por folhas de tetraedros de $\mathrm{SiO}_{4}$, ordenados de forma hexagonal e condensados com folhas octaédricas de hidróxidos de metais divalentes ou trivalentes [4]. Dentre as argilas do tipo três camadas (2:1), as montmorilonitas destacam-se, pois apresentam mais aplicações industriais que todas as outras argilas reunidas, sendo um material extremamente versátil e de perfil adequado para obtenção de produtos ou insumos de alto valor agregado [3, 7]. Estruturalmente as montmorilonitas pertencem ao grupo dos filossilicatos, caracterizadas por serem constituídas por uma folha de alumina octaédrica intercalada entre duas folhas de sílica tetraédricas opostas que são unidas entre si por forças eletrostáticas e ligação de hidrogênio [8]. Além disso, elas têm um excesso de carga superficial negativa devido à presença de substituições isomórficas não equivalentes dos átomos centrais nas camadas tetraédricas (por ex., $\mathrm{Al}$ por $\mathrm{Si}$ ) 
ou nas octaédricas (por ex., $\mathrm{Mg}$ por $\mathrm{Al}$ ). Consequentemente, a carga negativa pode interagir fortemente por forças eletrostáticas com cátions de balanceamento de carga, tipicamente íons de metais alcalinos, existentes no espaço intercalar [9]. Adicionalmente, as interações entre as duas folhas de sílica são muito fracas, o que permite que água e íons intercambiáveis entrem na sua estrutura [10]. Uma ilustração esquemática da estrutura em camadas das montmorilonitas é esboçada na Fig. 1.

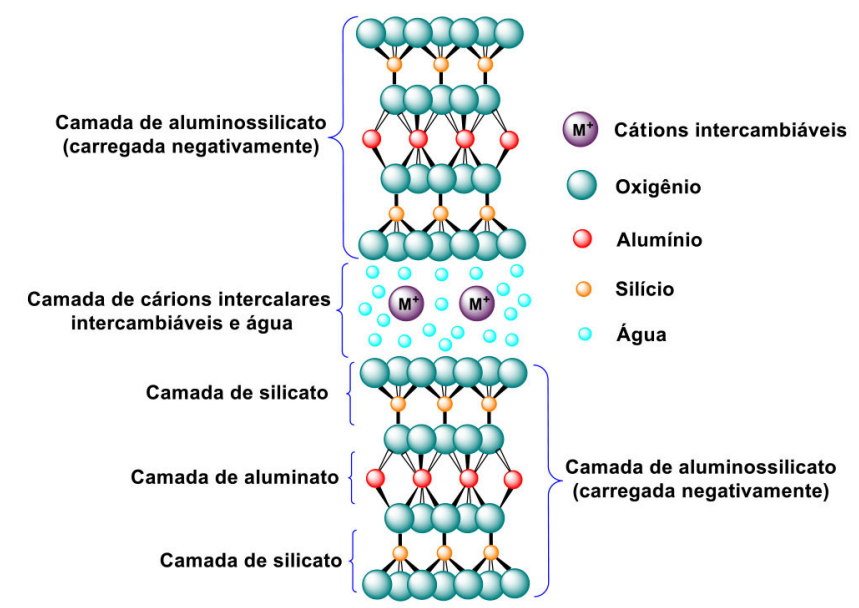

Figura 1: Estrutura em camadas das montmorilonitas (adaptada de [8]).

[Figure 1: Layered structure of montmorillonites (adapted from [8]).]

Durante as últimas décadas, as montmorilonitas vêm sendo extensivamente aplicadas nos mais variados setores industriais, a citar: na fabricação de materiais cerâmicos; adsorventes de contaminantes químicos em soluções aquosas; adsorventes em processos de clareamento na indústria têxtil e de alimentos; em processos de remediação de solos e em aterros sanitários; podem ser utilizadas no ajuste das propriedades reológicas de fluidos de perfuração de petróleo e de tintas; como carreadoras de moléculas orgânicas em cosméticos e fármacos; como catalisadores e também como suporte para catalisadores [1, 3, 11]. Essa versatilidade das montmorilonitas é devida, em parte, à sua capacidade de sofrer troca iônica levando a modificações importantes [2], o que de maneira indubitável leva a novos materiais de alto valor agregado. As argilas estão disponíveis naturalmente na superfície da terra de forma abundante, são consideradas atóxicas e não apresentam riscos ao homem e ao meio ambiente [12]. Adicionalmente, possuem excelentes propriedades de adsorção, grande potencial para permuta iônica e elevada área de superfície específica, apresentam disponibilidade comercial, baixo custo, fácil manipulação, não corrosividade e reutilizável [3, 5]. Em função desses atributos, o emprego das montmorilonitas como catalisadores ou promotores de reações orgânicas tem ganhado notoriedade no meio acadêmico durante os últimos anos [13-17]. O interesse dos químicos é traduzido pelos inúmeros relatos de sua utilização em reações orgânicas importantes como: alquilação [18] e acilação [19] de Friedel Crafts; nitração [20]; halogenação de compostos aromáticos [21]; reações de Diels-Alder [22]; reações de BaylisHillman [23]; rearranjo de Ferrier [24]; reações de BaeyerVilliger [25]; adição de Michael [26]; propagilação [27]; dentre outras [13-17]. De modo geral, as montmorilonitas mais aplicadas nestas transformações sintéticas são montmorilonita K10, montmorilonita KSF e tonsil, que são disponíveis comercialmente [28-30].

Diante deste cenário, o presente trabalho visou $o$ estudo das condições reacionais da reação de acetilação do 1,2:3,4-di- $O$-isopropilideno- $\alpha$-D-galactopiranose, empregando diferentes argilas, tais como: montmorilonita $\mathrm{K} 10$, montmorilonita KSF e tonsil. Embora a literatura descreva a reação de acetilação deste composto utilizando anidrido acético como doador do grupo acetóxi (OAc) juntamente com piridina [31], 1,4-diazabiciclo[2.2.2] octano [32], $\mathrm{Cu}\left(\mathrm{CH}_{3} \mathrm{CN}\right)_{4} \mathrm{OTf}$ [33], $\mathrm{Pd}(\mathrm{PhCN})_{2} \mathrm{Cl}_{2}$ [34], $\mathrm{MeSO}_{3} \mathrm{H}$ ou $\mathrm{SiO}_{2}-\mathrm{OSO}_{3} \mathrm{H}$ [35] e $\left(\mathrm{NH}_{4}\right)_{2} \mathrm{Ce}\left(\mathrm{NO}_{3}\right)_{6}$ [36], tais métodos apresentam algum tipo de limitação como, por exemplo: o uso de reagentes ou solventes tóxicos, corrosivos e de elevado custo; as reações são lentas; requerem a purificação do produto final; os catalisadores são de difícil recuperação; entre outras. É importante frisar que até o presente momento não há relatos de estudos biológicos para o 6-O-acetil1,2:3,4-di- $O$-isopropilideno- $\alpha$-D-galactopiranose. Esse fato engrandece ainda mais o presente estudo, pois a facilidade com que esse carboidrato é preparado neste trabalho pode motivar sua síntese e avaliação da atividade biológica. Sabese atualmente que os carboidratos são uma grande família de biomoléculas que possuem estruturas altamente diversas, estando presente na superfície e no interior das células principalmente na forma de glicoproteínas, proteoglicanos e glicolipídeos. Além disso, os carboidratos atuam em uma variedade de processos fisiológicos e patológicos através de interações com proteínas [37]. Nesse sentido, o estudo com essas biomoléculas é uma das áreas mais importantes em uma era pós-genômica.

\section{MATERIAIS E MÉTODOS}

Equipamentos, materiais, solventes e reagentes: em geral foram utilizados reagentes e solventes na sua forma comercial (P.A.), dos fornecedores Sigma-Aldrich, Vetec e Cinética. Os solventes utilizados foram purificados e secos conforme os protocolos Perrin e Armarego [38]; assim, o hexano e o acetato de etila (AcOEt) foram destilados utilizando coluna de Vigreux antes do uso, enquanto que a acetona foi destilada sob iodeto de sódio. O acompanhamento das reações foi realizado através de cromatografia em camada delgada (CCD). Para revelação dos compostos foi utilizada solução etanólica ácida (EtOH/ $\mathrm{H}_{2} \mathrm{SO}_{4}$ 9,5:0,50) sob aquecimento. Para cromatografia em coluna foi utilizada sílica-gel 60 (Merck, 70-230 mesh) como fase estacionária e sistemas hexano:acetato de etila como fase móvel (inicialmente 100\% de hexano até $70 \%$ de hexano e $30 \%$ de acetato de etila). Nos testes de reuso 
da argila foi utilizada uma centrífuga (Centribio, 802B) para decantar a argila e permitir a retirada do líquido sobrenadante. Os espectros de ressonância magnética nuclear foram registrados em um espectrômetro (Varian, Unity Plus) de $300 \mathrm{MHz}$. Os deslocamentos químicos foram expressos em ppm em relação ao pico residual do clorofórmio $(7,26 \mathrm{ppm})$ para os espectros de hidrogênio, em relação ao pico central do $\mathrm{CDCl}_{3}(77,0 \mathrm{ppm})$ para os espectros de carbono. Todas as constantes de acoplamento $(J)$ foram descritas em hertz $(\mathrm{Hz})$. Os pontos de fusão foram realizados no Electrothermal série IA 9100 Digital Melting Point. Os espectros de infravermelho foram registrados em um espectrofotômetro de IV com transformada de Fourier (Bruker, IFS66), com número de scans de 16, resolução 4, e número de onda de 4000 a $400 \mathrm{~cm}^{-1}$, sendo as amostras preparadas como filmes finos ou pastilhas de $\mathrm{KBr}$. As análises de rotação específica foram determinadas em um polarímetro digital (Jasco, P-2000) equipado com luz de sódio em comprimento de onda de $589 \mathrm{~nm}$.

Procedimento experimental. Preparação do 1,2:3,4-di$O$-isopropilideno- $\alpha$-D-galactopiranose (2): em um balão de fundo redondo, contendo $45 \mathrm{~mL}$ de propanona, foram adicionados D-galactose anidra $\mathbf{1}(1,8 \mathrm{~g}, 10,0 \mathrm{mmol}), \mathrm{CuSO}_{4}$ anidro $(4,0 \mathrm{~g}, 25 \mathrm{mmol})$ e $\mathrm{H}_{2} \mathrm{SO}_{4}$ concentrado $(0,23 \mathrm{~mL})$. A mistura reacional foi agitada em temperatura ambiente em atmosfera inerte. Uma vez comprovado o término da reação por $\mathrm{CCD}, \mathrm{o} \mathrm{CuSO}_{4}$ foi removido por filtração a vácuo e lavado com acetona a fim de extrair o máximo de produto que por ventura pudesse estar impregnado neste sal. Na sequência, o filtrado foi neutralizado com solução saturada de $\mathrm{NaHCO}_{3}$. O produto bruto foi rotaevaporado e purificado por cromatografia em coluna em um sistema eluente hexano/acetato de etila (75:25). Preparação do 6-O-acetil1,2:3,4-di-O-isopropilideno- $\alpha$-D-galactopiranose (3): em um balão de fundo redondo, contendo $1 \mathrm{~mL}$ de anidrido acético, foram adicionados $0,25 \mathrm{mmol}$ do composto 2 $(65 \mathrm{mg})$ e a montmorilonita K10 $(65 \mathrm{mg}, 100 \% \mathrm{~m} / \mathrm{m})$. A mistura reacional foi agitada por 7,5 min em temperatura ambiente. Após o término da reação a fase orgânica foi lavada com solução saturada de $\mathrm{NaHCO}_{3}(50 \mathrm{~mL})$ e seca com $\mathrm{MgSO}_{4}$. O produto bruto foi concentrado utilizando um evaporador rotativo e purificado por cromatografia em coluna em um sistema eluente hexano/acetato de etila (75:25). Procedimento para o reuso da montmorilonita K10: em um tubo de ensaio de $10 \mathrm{~mL}$, contendo 0,25 mmol do composto 2 (65 $\mathrm{mg})$ e anidrido acético $(1,0$ $\mathrm{mL}$ ), adicionou-se a montmorilonita K10 (65 mg, 100\% $\mathrm{m} / \mathrm{m}$ ) e agitou-se a mistura reacional em temperatura ambiente. Finalizada a reação, a mistura reacional foi centrifugada por $10 \mathrm{~min}$ a $1500 \mathrm{rpm}$. Após esse período, o tubo foi retirado da centrífuga e o líquido sobrenadante foi removido. Adicionou-se acetato de etila $(2,0 \mathrm{~mL})$ ao tubo e este foi centrifugado por mais 10 min e a fase orgânica foi removida. Esse último procedimento foi repetido por três vezes para garantir uma lavagem da montmorilonita e verificar a eficiência em reações subsequentes. Após essa lavagem reutilizou-se a argila em outros ciclos reacionais.

\section{RESULTADOS E DISCUSSÃO}

A reação de acetalização seletiva na D-galactose (1) utilizando acetona na presença de sulfato de cobre anidro e ácido sulfúrico é esquematizada na Fig. 2. O composto 2 foi obtido na forma de um líquido amarelado após $36 \mathrm{~h}$ de reação com rendimento de $83 \%$, sendo este resultado superior ao descrito na literatura [39]. Adicionalmente, a partir das informações disponíveis na literatura [40], o mecanismo para a reação de acetalização da D-galactose (1) é sugerido na Fig. 3. Vale ressaltar que foram obtidos 2,16 $\mathrm{g}(83 \%)$ do 1,2:3,4-di- $O$-isoproleno- $\alpha$-D-galactopiranose 2 , na forma de um óleo amarelado, e os dados espectroscópicos foram: $[\alpha]_{D}^{25}-44,3$ (c 1.0, MeOH); IV (pastilha de $\mathrm{KBr}$ ) $v_{\max } 3440,2984,2937,1702,1379,1212,1069 \mathrm{~cm}^{-1}$; RMN ${ }^{1} \mathrm{H}\left(400 \mathrm{MHz}, \mathrm{CDCl}_{3}\right) \delta 5,55\left(d, 1 \mathrm{H}, J_{3,4}=5,08 \mathrm{~Hz}, \mathrm{H}-3\right)$, $4,60\left(d d, 1 \mathrm{H}, J_{5,4}=2,36 \mathrm{~Hz}, J_{5,8}=7,84 \mathrm{~Hz}, \mathrm{H}-5\right), 4,32(d d$, $\left.1 \mathrm{H}, J_{4,3}=5,08 \mathrm{~Hz}, J_{4,5}=2,36 \mathrm{~Hz}, \mathrm{H}-4\right), 4,26\left(d d, 1 \mathrm{H}, J_{8.5}=\right.$ $\left.7,84 \mathrm{~Hz}, J_{8,9}=1,56 \mathrm{~Hz}, \mathrm{H}-8\right), 3,89-3,69$ ( $m, 3 \mathrm{H}, \mathrm{H}-9, \mathrm{H}-10$, $\mathrm{H}-10 '), 2,16(s l, 1 \mathrm{H}, \mathrm{OH}), 1,44\left(s, 3 \mathrm{H},-\mathrm{CH}_{3}\right), 1,32(s, 6 \mathrm{H}$, $\left.-\mathrm{CH}_{3}\right), 1,24\left(s, 3 \mathrm{H},-\mathrm{CH}_{3}\right)$; RMN ${ }^{13} \mathrm{C}\left(100 \mathrm{MHz}, \mathrm{CDCl}_{3}\right) \delta$ $109,4,108,6,96,2,71,5,70,7,70,5,68,1,62,3,29,2,26,0$, $25,9,24,3$.

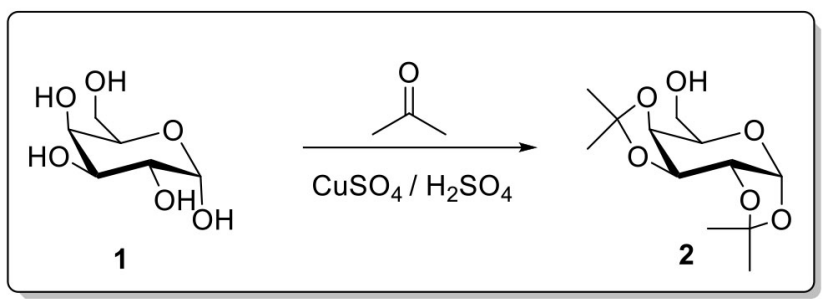

Figura 2: Esquema da reação de acetalização da D-galactose. [Figure 2: Scheme of the acetalization reaction of D-galactose.]

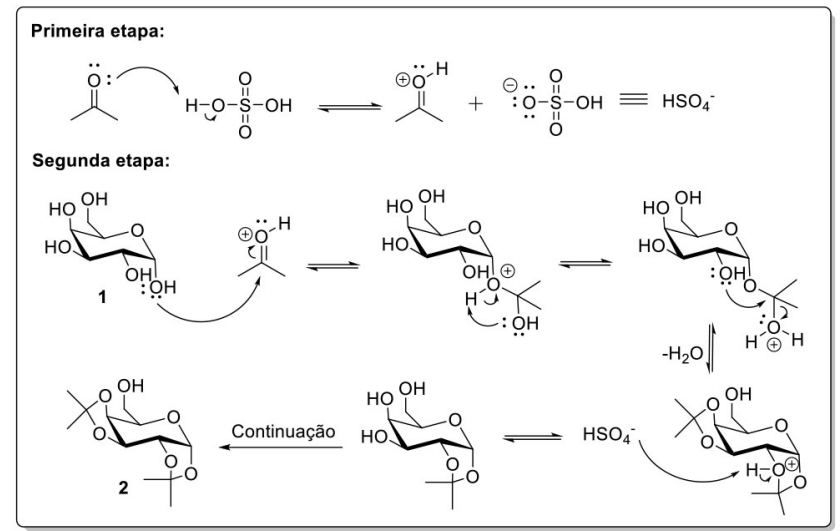

Figura 3: Proposta mecanística da acetalização da D-galactose. [Figure 3: Mechanistic proposal of the D-galactose acetalization.]

Para a otimização das condições reacionas da acetilação do composto $\mathbf{2}$, alguns parâmetros foram analisados, como o tipo e a quantidade de argila, assim como o seu próprio reuso. Inicialmente verificou-se a influência do tipo de argila, sendo utilizadas as argilas montmorilonita K10, montmorilonita KSF e tonsil. Os resultados desse estudo estão detalhados na Tabela I. Quando as montmorilonitas K10 e KSF foram 
Tabela I - Influência do tipo de argila utilizada na reação de acetilação do composto 2.

[Table I - Influence of the type of clay used in the acetylation reaction of the compound 2.]

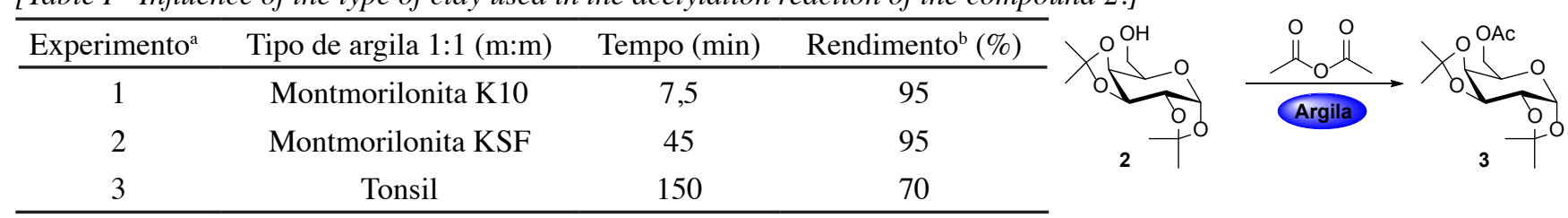

a - experimento utilizando 0,25 mmol do composto 2, 1,00 $\mathrm{mL}$ do anidrido acético e proporção de 1:1

(massa:massa) da argila com relação ao composto $2 ;^{b}$ - rendimento dos produtos isolados.

utilizadas na reação de acetilação, o produto 3 foi obtido em excelente rendimento (experimentos 1 e 2 ). Constatouse que a reação utilizando a argila tonsil levou ao produto 3, porém a reação apresentou um rendimento de $70 \%$ (experimento 3), mesmo deixando por um tempo reacional de $150 \mathrm{~min}$. A montmorilonita K10 apresentou melhor capacidade catalítica nesta reação, uma vez que o produto 3 foi obtido em menor intervalo de tempo e em excelente rendimento (experimento 1). Uma provável justificativa para o melhor desempenho da montmorilonita K10 nesta reação é devido a sua grande área superficial $\left(220-270 \mathrm{~m}^{2} \cdot \mathrm{g}^{-1}\right)$ quando comparada a montmorilonita KSF $\left(20-40 \mathrm{~m}^{2} \cdot \mathrm{g}^{-1}\right)$ [41], o que proporciona uma maior interação entre os substratos e seus sítios ácidos dessa argila.

Uma vez constatado que a melhor argila foi a montmorilonita K10, prosseguiu-se com o estudo da influência da quantidade desta argila na reação de acetilaçao do composto $\mathbf{2}$. Os resultados estão sumarizados na Tabela II. Os melhores resultados foram obtidos empregando as proporções $1,0,75$ e $0,5 \mathrm{~m} / \mathrm{m}$ da montmorilonita $\mathrm{K} 10$ (experimentos 3, 4 e 5), uma vez que levou ao produto $3 \mathrm{em}$ elevado rendimento e baixo tempo reacional. No entanto, quando as proporções 2,0 e $1,5 \mathrm{~m} / \mathrm{m}$ da montmorilonita K10 (experimentos 1 e 2) foram utilizados o produto 3 foi obtido, contudo observou-se a formação de subprodutos. Quando as quantidades de argila foram reduzidas para proporções 0,1 e $0,05 \mathrm{~m} / \mathrm{m}$ (experimentos 7 e 8 ) a reação não foi completa. Na ausência da montmorilonita K10 não houve formação do produto 3 . Levando em consideração o custo versus benefício da reação de acetilação do composto $\mathbf{2}$, a quantidade de montmorilonita K10 foi padronizada em 1:1 $\mathrm{m} / \mathrm{m}$, pois o produto 3 foi obtido com $95 \%$ de rendimento em 7,5 min. Uma proposta mecanística para a reação de acetilação do composto 2 é demonstrada na Fig. 4. Deste modo, a montmorilonita $\mathrm{K} 10$ ativou in situ o anidrido acético possibilitando uma maior deslocalização de carga tornando o carbono carbonílico mais eletrofílico, logo suscetível ao ataque nucleofílico do grupo hidroxila do composto 2 [42].

Cabe salientar que a acetilação do composto 2 não envolveu o uso de solvente orgânico, sendo esse um aspecto importante do método proposto, uma vez que há uma crescente preocupação dos efeitos nocivos dos solventes orgânicos no meio ambiente e no corpo humano. Nesse aspecto, as reações orgânicas operadas com solventes verdes ou sem solventes orgânicos convencionais despertaram a atenção dos químicos orgânicos e medicinais [43]. Além disso, a não utilização de solventes diminui os custos de
Tabela II - Influência da quantidade de montmorilonita K10 na reação de acetilação do composto 2 .

[Table II - Influence of the amount of montmorillonite K10 on the acetylation reaction of the compound 2.]

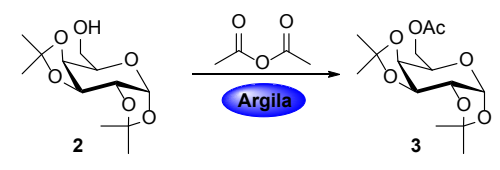

\begin{tabular}{|c|c|c|c|}
\hline Experimento $^{\mathrm{a}}$ & $\begin{array}{l}\text { Proporção } \\
(\mathrm{m} / \mathrm{m})\end{array}$ & $\begin{array}{l}\text { Tempo } \\
(\mathrm{min})\end{array}$ & $\begin{array}{c}\text { Rendimento }^{\mathrm{b}} \\
(\%)\end{array}$ \\
\hline 1 & 2,00 & 2,5 & 80 \\
\hline 2 & 1,50 & 4,0 & 81 \\
\hline 3 & 1,00 & 7,5 & 95 \\
\hline 4 & 0,75 & 10 & 91 \\
\hline 5 & 0,50 & 15 & 93 \\
\hline 6 & 0,25 & 17 & 86 \\
\hline 7 & 0,10 & 25 & 70 \\
\hline 8 & 0,05 & 100 & 60 \\
\hline 9 & $--^{c}$ & - & - \\
\hline
\end{tabular}

$\bar{a}$ - experimento utilizando 0,25 mmol do composto 2, 1,00 $\mathrm{mL} \mathrm{do}$ anidrido acético e proporção (massa:massa) da argila com relação ao composto $2 ;^{b}$ - rendimento dos produtos isolados; ${ }^{c}$ - experimento isento de argila.

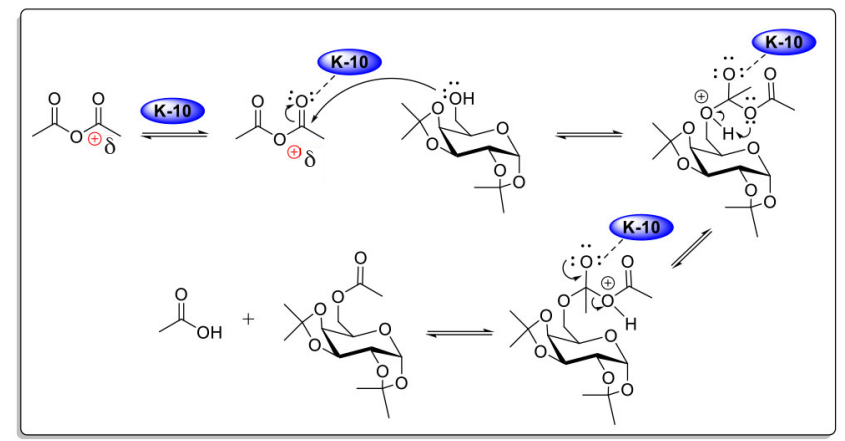

Figura 4: Proposta mecanística para a reação de acetilação promovida pela montmorilonita $\mathrm{K} 10$.

[Figure 4: Mechanistic proposal for the acetylation reaction promoted by montmorillonite K10.]

produção, tornando os processos mais simples e econômico. Outro aspecto importante foi que a montmorilonita K10 atuou como catalisador quimiosseletivo, uma vez que o composto 2 foi acetilado e não houve remoção dos grupos cetais presentes em sua estrutura, diferentemente do que 
aconteceria se fosse utilizado ácido sulfúrico ou ácido clorídrico, frequentemente utilizados na reação de acetilação $[44,45]$. É importante destacar que a estrutura do produto 3 foi confirmada por ressonância manética nuclear de ${ }^{1} \mathrm{H} \mathrm{e}{ }^{13} \mathrm{C}$. No espectro de RMN ${ }^{1} \mathrm{H}$ (Fig. 5), foram observados quatro simpletos em campo alto na região entre 1,33 a 1,51 ppm que corresponderam aos hidrogênios metílicos H-1, H-2, H-5 e H-6. O simpleto em 2,08 ppm foi atribuído aos hidrogênios metílicos do grupo -OAc, sendo este sinal a evidência da reação de acetilação. $\mathrm{O}$ sinal atribuído ao hidrogênio H-3 apresentou uma multiplicidade do tipo dupleto (expansão) em 5,53 ppm devido ao acoplamento com H-4 $\left(J_{3,4}=5,1\right.$ $\mathrm{Hz})$, que por sua vez também acopla com H-5 $\left(J_{4,3}=5,1 \mathrm{~Hz}\right.$; $J_{4,5}=2,4 \mathrm{~Hz}$ ) originando um dupleto de dupleto (expansão)

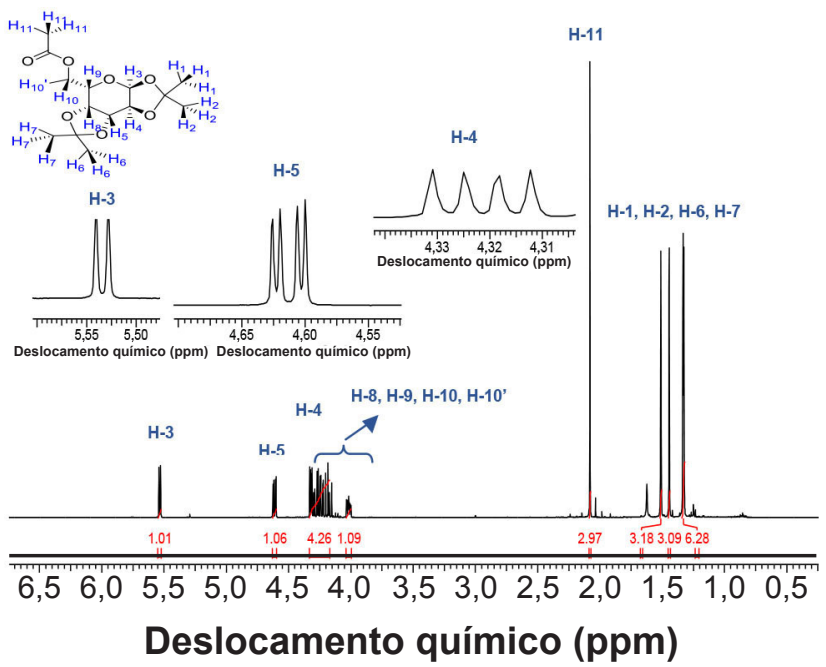

Figura 5: Espectro de $\mathrm{RMN}{ }^{1} \mathrm{H}\left(\mathrm{CDCl}_{3}, 400 \mathrm{MHz}\right)$ do composto 3. [Figure 5: ${ }^{1} \mathrm{H}$-NMR spectrum $\left(\mathrm{CDCl}_{3}, 400 \mathrm{MHz}\right)$ of compound 3.]

com deslocamento químico de 4,32 ppm. O sinal em 4,6 ppm correspondeu ao hidrogênio H-5, o qual acoplou com $\mathrm{H}-4$ e H-8 na forma de um dupleto de dupleto $\left(J_{5,4}=2,3 ; \mathrm{Hz}\right.$; $J_{5,8}=7,8 \mathrm{~Hz}$ ). Os demais hidrogênios e seus respectivos sinais estão sinalizados na Fig. 5.

Analisando o espectro de $\mathrm{RMN}{ }^{13} \mathrm{C}$ do produto 3 verificou-se a ocorrência de 14 sinais, quantidade igual à de carbonos presente na estrutura deste composto. Os sinais em campo alto que se estenderam na região entre 20,9 a 25,9 ppm foram atribuídos aos carbonos metílicos, pois estes se encontraram em um ambiente de maior densidade eletrônica. Por outro lado, o sinal em campo baixo com deslocamento químico de 170,9 ppm correspondeu ao carbono carbonílico do grupo -OAc (desblindado devido ao efeito de anisotropia diamagnética da ligação $\mathrm{C}=\mathrm{O}$ ), o que corroborou a estrutura do produto 3 (Fig. 6). O 6-O-acetil-1,2:3,4-di- $O$-isopropilideno$\alpha$-D-galactopiranose 3 foi obtido na forma de um sólido branco (P.F. 109-110 ${ }^{\circ} \mathrm{C}$ ), onde seus dados espectroscópicos foram: $[\alpha]_{D}^{25}-44,1$ (c 1.0, MeOH); IV (pastilha de $\mathrm{KBr}$ ) $v_{\text {max }}$ 2990, 2961, 2902, 1740, 1376, 1249, 1072, $1005 \mathrm{~cm}^{-1}$; RMN ${ }^{1} \mathrm{H}\left(400 \mathrm{MHz}, \mathrm{CDCl}_{3}\right) \delta 5,55\left(d, 1 \mathrm{H}, J_{34}=5,08 \mathrm{~Hz}, \mathrm{H}-3\right), 4,61$ $\left(d d, 1 \mathrm{H}, J_{5,4}=2,36 \mathrm{~Hz}, J_{5,8}=7,80 \mathrm{~Hz}, \mathrm{H}-5\right), 4,32\left(d d, 1 \mathrm{H}, J_{4,3}=\right.$

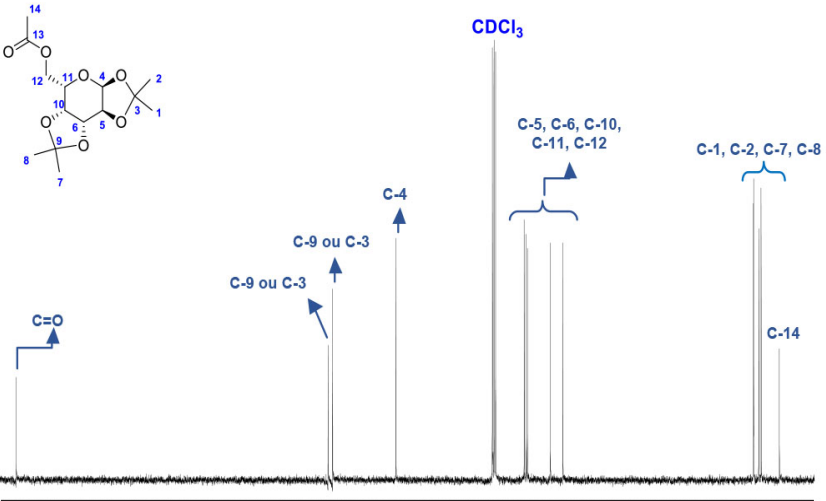

$16816015214413612812011210496 \quad 88 \quad 8072 \quad 64 \quad 56 \quad 48 \quad 40 \quad 32 \quad 24 \quad 16$ Deslocamento químico (ppm)

Figura 6: Espectro de $\mathrm{RMN}{ }^{13} \mathrm{C}\left(\mathrm{CDCl}_{3}, 100 \mathrm{MHz}\right)$ do composto 3 . [Figure 6: ${ }^{13} \mathrm{C} \mathrm{NMR}$ spectrum $\left(\mathrm{CDCl}_{3}, 100 \mathrm{MHz}\right)$ of compound 3.]

$\left.5,08 \mathrm{~Hz}, J_{4,5}=2,36 \mathrm{~Hz}, \mathrm{H}-4\right), 4,23\left(d d, 1 \mathrm{H}, J_{8,5}=7,84 \mathrm{~Hz}, J_{8,9}=\right.$ $1,96 \mathrm{~Hz}, \mathrm{H}-8), 4,2-4,0$ ( $m, 3 \mathrm{H}, \mathrm{H}-9, \mathrm{H}-10, \mathrm{H}-10$ '), 2,1 ( $s, 3 \mathrm{H}$, -OAc), 1,51 (s, 3H, - $\left.\mathrm{CH}_{3}\right), 1,44\left(s, 3 \mathrm{H},-\mathrm{CH}_{3}\right), 1,33(s, 3 \mathrm{H}$, $\left.-\mathrm{CH}_{3}\right), 1,32\left(s, 3 \mathrm{H},-\mathrm{CH}_{3}\right)$; RMN ${ }^{13} \mathrm{C}\left(100 \mathrm{MHz}, \mathrm{CDCl}_{3}\right) \delta$ $170,9,109,6,108,0,96,3,71,1,70,7,70,4,65,9,63,4,26,0$, $25,9,24,5,20,9$.

Por fim, foi avaliado por quantos ciclos reacionais a montmorilonita K10 era capaz de promover a reação de acetilação sem perder sua eficiência. Para tanto, realizaramse vários ciclos reacionais e ao final de cada ciclo o líquido sobrenadante foi removido. Em seguida, a argila foi lavada com acetato de etila antes do novo ciclo. Os resultados estão descritos na Tabela III. A montmorilonita K10 manteve-se eficiente por três ciclos reacionais e praticamente não houve alterações no tempo reacional e no rendimento (experimentos 1-4). Observou-se que a partir do quarto ciclo a eficiência da montmorilonita K10 diminuiu, uma vez que o tempo dobrou e o rendimento máximo obtido foi de $72 \%$ (experimento 5), provavelmente devido à saturação dos sítios ácidos presentes na montmorilonita K10. De modo geral, estes resultados

Tabela III - Estudo do reuso da montmorilonita K10 na acetilação do composto 2 .

[Table III - Study of the reuse of montmorillonite K10 in the acetylation of compound 2.]

\begin{tabular}{cccc}
\hline Experimento $^{\mathrm{a}}$ & Ciclo & $\begin{array}{c}\text { Tempo } \\
(\mathrm{min})\end{array}$ & $\begin{array}{c}\text { Rendimento }^{\mathrm{b}} \\
(\%)\end{array}$ \\
\hline 1 & - & 7,5 & 95 \\
2 & 1 & 7,5 & 96 \\
3 & 2 & 7,5 & 95 \\
4 & 3 & 7,5 & 94 \\
5 & 4 & $15^{\mathrm{c}}$ & 72 \\
6 & 5 & 30 & 70 \\
\hline
\end{tabular}

a - experimento utilizando 0,25 mmol do composto 2, 1,00 $\mathrm{mL}$ do anidrido acético e proporção $1(\mathrm{~m} / \mathrm{m})$ da argila com relação ao composto $2,{ }^{b}$ - rendimento dos produtos isolados; ${ }^{c}$ - aumento do tempo no quarto ciclo reacional devido, provavelmente, à saturação dos sítios ácidos presentes na estrutura da K10. 
foram muitos satisfatórios, pois a montmorilonita K10 promoveu a acetilação do composto 2 em um curto tempo reacional e, além disso, foram obtidos excelentes rendimentos. Adicionalmente, essa argila pode ser utilizada por três ciclos reacionais sem perda de eficiência.

\section{CONCLUSÕES}

Neste trabalho é descrito um novo método para promover a reação de acetilação do 1,2:3,4-di- $O$-isopropilideno$\alpha$-D-galactopiranose utilizando como catalisador a montmorilonita K10. O método demonstrou ser altamente eficiente e quimiosseletivo, uma vez que não houve perda dos grupos cetais durante a acetilação do 1,2:3,4-di- $O$ isopropilideno- $\alpha$-D-galactopiranose. $\mathrm{O}$ produto $6-O$-acetil 1,2:3,4-di- $O$-isopropilideno- $\alpha$-D-galactopiranose foi obtido em curto tempo $(7,5 \mathrm{~min})$ e em excelente rendimento (95\%), salientando que o método apresenta eficiência energética. A acetilação do 1,2:3,4-di- $O$-isopropilideno$\alpha$-D-galactopiranose aconteceu em condições isentas de solventes e, como a montmorilonita K10 é um reagente barato, de fácil manipulação, comercialmente disponível e compatível com o meio ambiente (atóxica e não corrosiva), este método de reação poderia ser adotado em uma escala comercial. Também se observou que essa argila manteve sua atividade catalítica por três ciclos reacionais sem perder eficiência.

\section{AGRADECIMENTOS}

Os autores agradecem às agências de fomento $\mathrm{CNPq}$ (447361/2014-7) ao PRONEM/FACEPE (APQ-04761.06/14) pelo suporte financeiro, à CAPES pelas bolsas concedidas e a Central Analítica do DQF-UFPE pelas análises dos compostos.

\section{REFERÊNCIAS}

[1] S.O. Obaje, J.I. Omada, U.A. Dambatta, Int. J. Sci. Technol. 3 (2013) 264.

[2] R. Zhu, Q. Chen, Q. Zhou, Y. Xi, J. Zhu, H. He, Appl. Clay Sci. 123 (2016) 239.

[3] A.R.V. Silva, H.C. Ferreira, Rev. Eletr. Mater. Proc. 32 (2008) 26.

[4] A.C.V. Coelho, P. de S. Santos, Quim. Nova 30 (2007) 146.

[5] E.H. Nickel, Can. Mineral. 33 (1995) 689.

[6] B. Velde, Composition and mineralogy of clay minerals: origin and mineralogy of clays, Springer, Berlin (1995).

[7] M.K. Uddin, Chem. Eng. J. 308 (2017) 438.

[8] M.F. Brigatti, E. Galan, B.K.G. Theng, Structure and mineralogy of clay minerals, developments in clay science, vol. 5A, Elsevier Press, United States (2013).

[9] J. Liua, G. Zhang, Phys. Chem. Chem. Phys. 16 (2014) 8178.

[10] E. Fjar, R.M. Holt, A.M. Raaen, R. Risnes, P. Horsrud, Petroleum related rock mechanics, developments in petroleum science, $2^{\text {nd }}$ ed., vol. 53, Elsevier Press, United States (2008).

[11] E. Teixeira-Neto, A.A. Teixeira-Neto, Quim. Nova 32 (2009) 809.

[12] G. Crini, P.M. Badot, Sorption process and pollution, conventional and non-conventional sorbents for pollutant removal from wastewaters, Presses Un. Franche-Comté, France (2010).

[13] F.P.R. Crisostomo, R. Carrillo, T. Martin, V.S. Martin, Tetrahedron Lett. 46 (2005) 2829.

[14] D. Sujaya, B. Török, Org. Prep. Proced. Int. 40 (2008) 1.

[15] S. Naskar, P. Paira, S. Mondal, A. Maity, A. Hazra, Tetrahedron 66 (2010) 5196.

[16] E. Kumaran, M. Santhi, K.K. Balasubramanian, S. Bhagavathy, Carbohydr. Res. 346 (2011) 1654.

[17] S.B. Bharate, A.K. Padala, B.A. Dar, R.R. Yadav, B. Singh, R.A. Vishwakarma, Tetrahedron Lett. 54 (2013) 3558.

[18] V. Singh, A. Khurana, I. Kaur, V. Sapehiyia, G.L. Kad, J.J. Singh, Chem. Soc. Perkin Trans. 1 (2002) 1766.

[19] F. Bigi, M.L. Conforti, R. Maggi Sartori, Tetrahedron 56 (2000) 2709.

[20] B.M. Choudary, M. Sateesh, M.L. Kantam, K.K. Rao, K.V.R. Prasad, K.V. Raghavan, J.A.R.P. Sarma, Chem. Commun. 0 (2000) 25.

[21] S. Perumal, V. Vijayabaskar, V. Gomathi, S. Selvaraj, Indian J. Chern. 38B (1999) 603.

[22] M.R. Dintzner, A.J. Little, M. Pacilli, D.J. Pileggi, Z.R. Osner, T.W. Lyons, Tetrahedron Lett. 48 (2007) 1577.

[23] P. Shanmugam, P. Rajasingh, Tetrahedron 60 (2004) 9283.

[24] J.R. De Freitas Filho, R.M. Srivastava, Y. Soro, L. Cottier, G.J. Descotes, Carbohyd. Chem. 20 (2001) 561.

[25] T. Kawabata, N. Fujisaki, T. Shishido, K. Nomura, T. Sano, K. Takehira, J. Mol. Catal. A Chem. 253 (2006) 279.

[26] N.S. Shaikh, V.H. Deshpande, A.V. Bedekar, Tetrahedron 57 (2001) 9045.

[27] J.J.R. Freitas, T.R. Couto, I.H. Cavalcanti, J.C.R. Freitas, Q.P.S. Barbosa, R.A. Oliveira, Tetrahedron Lett. 57 (2016) 760.

[28] S. Muthumariappan, A.U.J. Devi, Nanosci. Tech. 2 (2016) 227.

[29] R. Miranda, O.V. Vázquez, C.A.M. Vega, I.N. Vázquez, Y.M.V. Rodriguez, J.A.M. Serna, E.G. Ríos, M. Salmón, Molecules 18 (2013) 12820.

[30] M. Badali, J. Khalafy, M. Aghazadeh, R.H. Prager, Bull. Chem. Soc. Ethiop. 30 (2016) 129.

[31] Y. Sawama, Y. Yabe, H. Iwata, Y. Fujiwara, Y. Monguchi, H. Sajiki, Chem. Eur. J. 18 (2012) 16436.

[32] R. Ch, M. Tyagi, P.R. Patil, K.P.R. Kartha, Tetrahedron Lett. 52 (2011) 5841.

[33] E.A. Mensah, L. Earl, Catalysts 7 (2017) 33.

[34] E.A. Mensah, F.R. Reyes, E.S. Standiford, Catalysts 6 (2016) 27.

[35] S.K. Giria, K.P.R. Kartha, RSC Adv. 5 (2015) 11687.

[36] M. Seepersaud, S. Seecharan, L.J. Lalgee, N.K. Jalsa, 
Synth. Commun. 47 (2017) 853.

[37] I. Shin, K.S. Kim, Chem. Soc. Rev. 42 (2013) 4267.

[38] D.D.Perrin, W.L.F. Amarego, Purification of laboratory chemicals, $3^{\text {rd }}$ ed., Pergamon Press, Oxford (1996).

[39] O.T. Schmidt, Methods Carbohydr. Chem. 2 (1963) 318.

[40] M.V.N. Souza, Síntese orgânica: baseado em substâncias bioativas, Ed. Átomo, Campinas (2010). [41] J.Q. Jiang, Z. Zeng, Chemosphere 53 (2003) 53.
[42] R.N. Oliveira, A.L. Xavier, B.M. Guimarães, V.N.E. Melo, W.O. Valença, W.S. Nascimento, P.L.F. Costa, C.A. Camara, J. Chil. Soc. 59 (2014) 2610.

[43] T. Welton, Proc. R. Soc. A 471 (2015) 1.

[44] K. Danielmeier, E. Steckhan, Tetrah. Asymmetry 6 (1999) 1181.

[45] A.G. Cioletti, R.J. Alves, J.D. Souza Filho, J.G. Chaves, M.A.F. Prado, Synth. Commun. 30 (2000) 2019.

(Rec. 20/12/2017, Rev. 20/04/2018, Ac. 24/06/2018) 